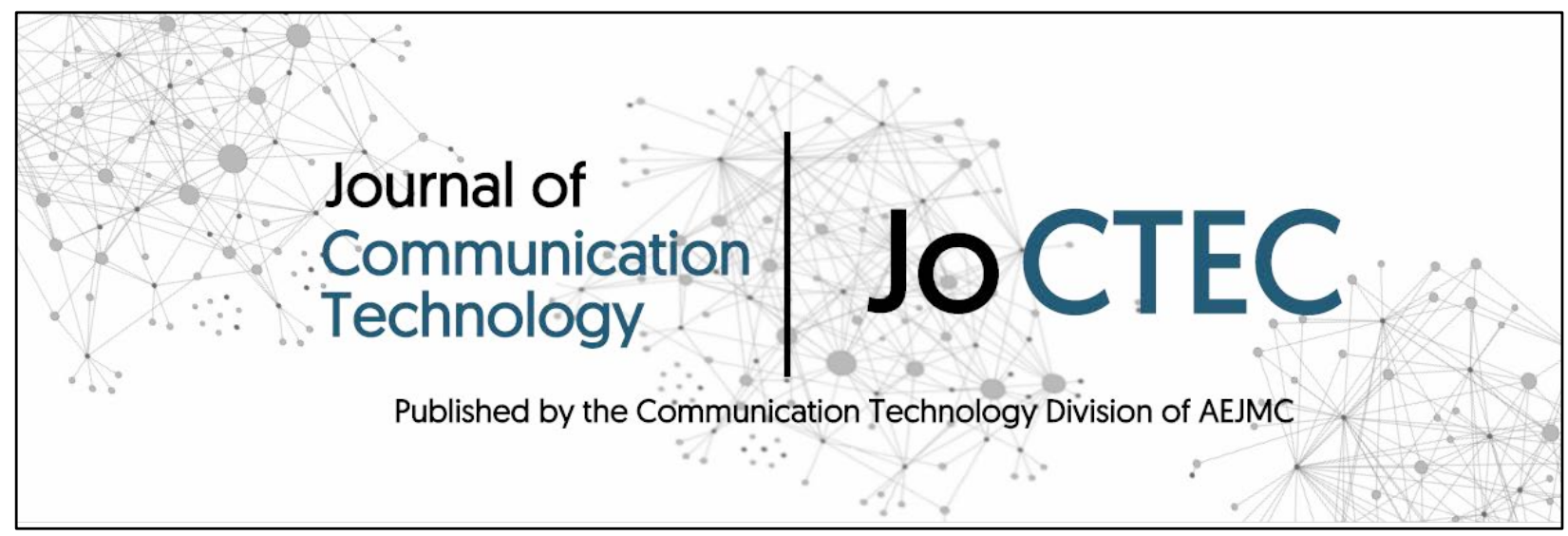

No, you probably don't touch your phone 2,617 times per day: A rationale for the Journal of Communication Technology

\author{
Dr. Jacob Groshek \\ Associate Professor of Emerging Media Studies \\ College of Communication \\ Boston University
}

Correspondence:

$617-353-6421$

jgroshek@bu.edu

Original manuscript accepted for publication in Journal of Communication Technology

Published by the Communication Technology Division of the Association for Education in Journalism and Mass Communication

DOI: 10.51548/joctec-2018-001 


\section{No, you probably don't touch your phone 2,617 times per day: A rationale for the Journal of Communication Technology}

Some statistics reach into popular culture, and they often become benchmarks by which both the general public and media researchers come to know and understand ourselves and our social world. One such statistic is how about two-thirds of people receive "at least some of their news" from social media, which comes from the venerable Pew Research Center (Shearer \& Gottfried, 2017) and is cited by many studies attempting to show how important social media is in relation to news. Of course, what is not reported in this instance is that the same report identifies only two-in-ten who report doing so often.

The point here is not to criticize Pew or those that cite that particular finding, but to prod deeper into how we know the things that (we think) we know. Which brings me to the statistic that is being reported now somewhat regularly that "we touch our phones . . a about 2,617 times a day" (Winnick \& Zolna, 2016).

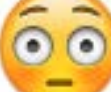

As reported, this finding includes the astonished emoji (above) and is from the firm known as dscout (see https://blog.dscout.com/mobile-touches). I've heard this figure mentioned at leading research events by some of the brightest folks working in the field, and, as is sometimes the case, an audience full of similarly smart and motivated scholars express their astonishment, nod, and move on thinking we've all learned something. But have we? Do weexperts in the area of digital, social, mobile, and online media-really know what we think we know? I don't know.

When I casually mentioned this figure to my Boston University colleague Jim Cummings over lunch recently, he (politely and rightly) scoffed and did some quick math, suggesting that 
the figure of about 2,600 touches would equate to over 100 touches per hour if one were to be awake 24 hours everyday. That impossibility led us to discuss what constituted a 'touch' (honestly, I didn't know) and how that would be humanly possible.

So, like any good social scientist, I started to investigate the claim further. Turns out that dscout considered a 'touch' to be every tap, type, swipe and click, gathered via an app. Beyond that, what I found was that it was based on just 94 Android users over a period of 5 days, but as best as I could tell, only two-thirds of those participated all five days. The others participated only 2 to 4 days. This means we are making claims about an entire population based on just 63 opt-in individuals.

More than that, when I did some additional simple math, I determined that, based on 18 hours spent awake, the figure of 2,617 touches per day add up to just over 2.4 mobile phone touches per minute. Of every waking minute. Of every day. Generally speaking, I'd say this figure is, as constructed, not impossible but also not very plausible - and that those 'touches' might not be meaningful indicators of 'interaction' between users or content. So, as I see it, the role of the Journal of Communication Technology is to be a space that facilitates discussion and cultivates understanding of the ways in which communication technologies are changing not only media processes and content, but also audiences, institutions, and society at large.

Going back to 2010 when Marcus Messner and Homero Gil de Zuñiga and I started on this adventure to publish our own journal for the Communication Technology Division of AEJMC, it seemed so simple and obvious that we needed another point of entry to tackle challenging problems germane to the field but that had broader implications as well. What can I say other than we were all young, ambitious, and perhaps just a bit too optimistic about what the process of launching a journal would entail? Though it has taken some eight-odd years of bureaucracy and countless hours of effort, and we are more or less published by default of out Boston University and the dedicated support of my research assistants, I am pleased to present 
the inaugural issue of JOCTEC, and happy to report that the relationship of us three founding editors is still intact, and perhaps even more solidified as a result.

Looking at this inaugural issue in particular, I believe we have articles that address issues of great importance as well as useful research and insights for our audience of scholars working in this area. For example, in our lead article, Dr. James Ivory highlights important approaches to methodological reform in media effects research in relation to communication technology. Along these lines, Dr. Erik Bucy makes an important contribution in terms of calibration, and how that can be managed among researchers working in areas of asymmetric message flows, particularly as this process plays out for the vast majority of what can be considered average users. Dr. Marcia DiStaso then provides some excellent examples of communication challenges in cybersecurity as well as pathways forward. Next, Dr. Richard Shaefer outlines key aspects of the integration between data visualization and the capacity to analyze and understand social trends. Finally, Wasim Ahmed provides an essential overview of tools for doing social media research in the contemporary era that will benefit anyone interested in this area of study.

And, to reiterate from our call for papers, since communication technologies themselves have now come to fulfill a central, social role in virtually all forms of mediated communication, the journal welcomes scholarship from a broad area of inquiry. Provided that the focus pertains to communication technologies, this area of inquiry includes but is not limited to studies of advertising, science, networks, health, politics, history, policy, public relations, management, economics, ethics, minorities, visual communication, and social media.

In addition, as the journal is vitally positioned in a growing international field, it strives to be a home for all theoretical and methodological perspectives. Research that informs debates from comparative empirical perspectives is especially welcome, though more conceptual and theoretical approaches are equally invited. Altogether, systematic and rigorous scholarship of 
communication technologies and their impacts from virtually any approach from micro to macro and throughout sub-disciplines will be considered.

It is with great pleasure I put forth this inaugural issue and look forward to growing the Journal of Communication Technology further as a collaborative effort with our community of contributors and readers. I am deeply indebted to the authors of this issue as well as the steadfast support of our esteemed editorial board. Thank you all, and please share our opensource publications widely.

If you are already reading through this issue, or have learned of JoCTEC through another means, we hope you will consider publishing with us. We offer not only expert review (as signified by our Editorial Board) but also fast turnaround and flexibility with content and format. Any questions, just ask. We will do our best to innovate and accommodate quality work that makes a valuable contribution to this field regardless the format.

We are off, with my profound thanks for your support! I look forward to many future successes with this initiative, and could not do it without the CTEC community.

\section{References}

Shearer, E., \& Gottfried, J. (2017, September 7). News use across social media platforms. Pew Research Center. Retrieved from http://www.journalism.org/2017/09/07/news-useacross-social-media-platforms-2017/

Winnick, M., \& Zolna, R. (2016, June 16). Putting a finger on our phone obsession; Mobile touches: A study on humans and their tech. Dscout. Retrieved from https://blog.dscout.com/mobile-touches 